\title{
NON-PRISMATIC BEAMLIKE STRUCTURES WITH 3D CROSS-SECTIONAL WARPING
}

\author{
G. MIGLIACCIO 1 \\ ${ }^{1}$ Department of Civil and Industrial Engineering, University of Pisa \\ Largo Lucio Lazzarino 2, 56122, Pisa, Italy \\ giovanni.migliaccio.it@gmail.com
}

Key words: tapered beams, cross-sectional warping, large displacements.

\begin{abstract}
Many complex engineering structures, e.g. blades of wind turbines and helicopters, are beamlike and non-prismatic. They may be tapered, twisted and curved in their unstressed state, undergo large displacements of the centre-line, and cross-sectional warping in and out of plane. For their structural modeling, an approach based on beam elements can be the best compromise between computational efficiency and accuracy, but classical beam models (see, for example, the monumental Love's treatise) may not be sufficient. Better results may be obtained by exploiting geometrically exact and asymptotic approaches. This paper proposes a physical-mathematical model for the aforementioned non-prismatic structures. Analytical results obtained for small warping and strain fields are presented and compared to the results obtainable from nonlinear 3D-FEM analyses.
\end{abstract}

\section{INTRODUCTION}

Beamlike structures, e.g. helicopter blades, wind blades, components of civil buildings and bridges, are widespread in engineering applications. Such structures may be tapered, twisted, and even curved in their unstressed state, have fully deformable transverse cross-sections, and undergo large displacements. For their structural modelling the best compromise between computational efficiency and accuracy can be obtained via schematizations based on suitable beam elements [1-3]. However, classical beam models for extension, twisting, bending and shear deformation, e.g. [4-6], may not be sufficient. Models based on geometrically exact and asymptotic approaches can provide better results [7]. Over the years several models have been proposed for beamlike bodies [8-13], and most of them are also summarized in reviews (e.g. [14-16]). Nevertheless, non-prismatic cases still require investigation. Generally speaking, the geometry of the non-prismatic beamlike body must be appropriately described, as the taper, twist, and curvature are important geometric features and should be explicitly included in the model. Moreover, the analysis should not be restricted to small displacements. The model should provide the stress and strain fields in the three-dimensional body, be rigorous and application-oriented, and provide classical results for prismatic cases. Following such main guidelines, a physical-mathematical model for the aforementioned structures is proposed in this work. Specifically, this paper addresses the mechanical modelling of non-prismatic beamlike elements subjected to large displacements of the centre-line's points, cross-sectional 
warping in and out of plane, and small strain, with particular focus on the effects of important geometric features, such as the cross-sectional taper. A model suitable for the problem at hand is introduced in section 2. Analytical results obtained by its application are presented in section 3. Numerical examples and comparisons with the results from nonlinear 3D-FEM simulations are finally shown in section 4.

\section{MECHANICAL MODEL}

An important point in modeling structures subject to large displacements is the description of their motion [17-22]. We describe a beamlike structure as a collection of deformable plane figures (transverse cross-sections) along a 3D curve (beam's centre-line). The displacement from the reference to the current state of each cross-sectional point consists of a global rigid motion onto which a local warping motion is superposed. In this way, the cross-sectional local motion can be examined independently of the global motion, and it is possible to consider the global motion to be large, while the local warping motion and the strain may be small.

\subsection{Kinematics and strain measures}

We begin introducing two local triads of orthogonal unit vectors. The first is the reference local triad, $b_{i}$, with $b_{1}$ tangent to the reference centre-line. It is a function of the reference arclength $s$, i.e. $b_{i}=b_{i}(s)$. The second triad, $a_{i}$, is an image of $b_{i}$ in the current state. It depends on the arc-length $\mathrm{s}$ and time $\mathrm{t}$, i.e. $\mathrm{a}_{\mathrm{i}}=\mathrm{a}_{\mathrm{i}}(\mathrm{s}, \mathrm{t})$. In general, the orientation of $\mathrm{a}_{\mathrm{i}}$ and $\mathrm{b}_{\mathrm{i}}$ relative to $\mathrm{a}$

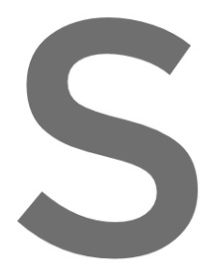

fixed rectangular franie, $\mathrm{c}_{\mathrm{i}}$, can be defined as

where $\mathrm{A}$ and $\mathrm{B}$ are proper orthogonal tensor fields. Figure current states of the structure in
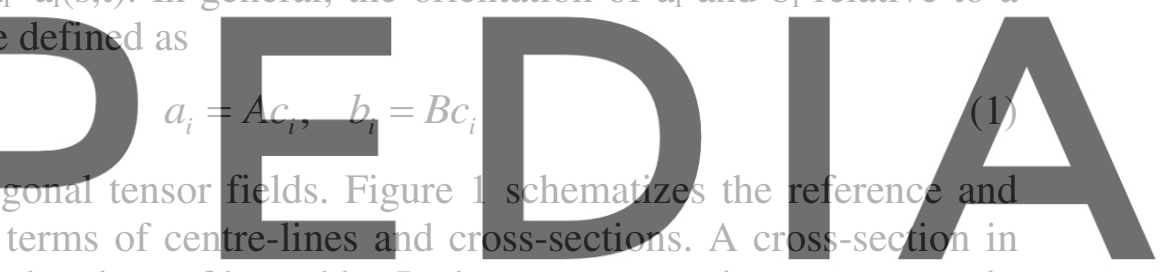

the reference state is contained in the plane of $b_{2}$ and $b_{3}$. In the current state it may not remain

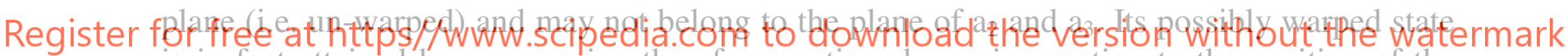
is in fact attained by superposing the aforementioned warping motion to the position of the points of the un-warped cross-section (as shown in Figure 1, right).

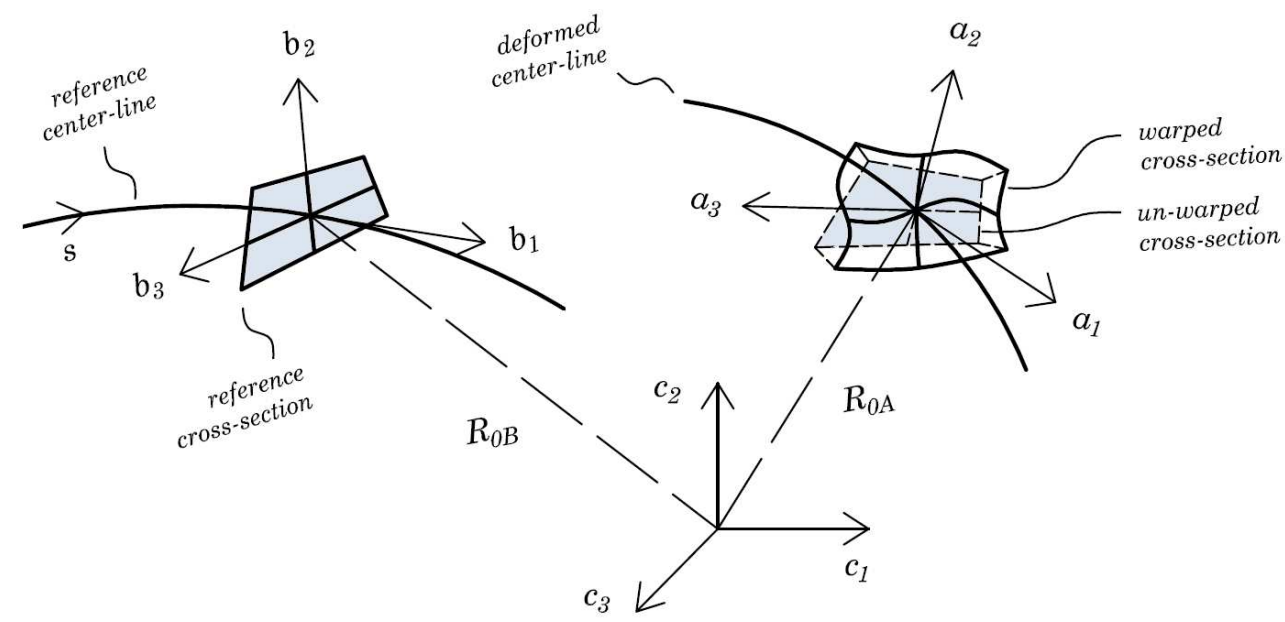

Figure 1: Schematic of reference and current states in terms of centre-lines, cross-sections and local frames 
Two mapping functions, $\mathrm{R}_{\mathrm{A}}$ and $\mathrm{R}_{\mathrm{B}}$, identify the positions of the structure's points in the current and reference states, respectively. The reference mapping function is

$$
R_{B}\left(z_{i}\right)=R_{0 B}\left(z_{1}\right)+x_{\alpha}\left(z_{i}\right) b_{\alpha}\left(z_{1}\right)
$$

where $\mathrm{R}_{0 \mathrm{~B}}$ provides the position of the reference centre-line relative to triad $c_{i}, b_{\alpha}$ are vectors of the reference local triad in the plane of the reference cross-section, $\mathrm{x}_{\alpha}$ identify the position of such cross-section's points relative to the reference centre-line, and $z_{i}$ are three independent mathematical variables which do not depend on time. More precisely, $z_{1}$ is equal to the reference arc-length $\mathrm{s}$, while $\mathrm{z}_{\alpha}$ belong to a bi-dimensional domain which is used to map the positions, $\mathrm{x}_{\alpha}$, of the cross-sections points.

Throughout this paper, Greek indices take values 2 and 3, Latin indices assume values 1, 2 and 3 , and repeated indices are summed over their range.

It is worth noting that $\mathrm{x}_{\mathrm{k}}$ may or may not be equal to $\mathrm{z}_{\mathrm{k}}$, depending on the modeling approach adopted and the structure to be modeled. Here we choose relations between $\mathrm{x}_{\mathrm{k}}$ and $Z_{k}$ to explicitly model the shape of the non-prismatic beams considered in this work. In particular, the spanwise variation of the cross-sectional shapes is modeled via the map

$$
x_{i}=\Lambda_{i j} z_{j}
$$

where $\Lambda_{i j}$ are functions of $\mathrm{z}_{1}$. We consider curved and twisted beamlike bodies with bi-tapered transverse cross-sections, with map (3) reducing to

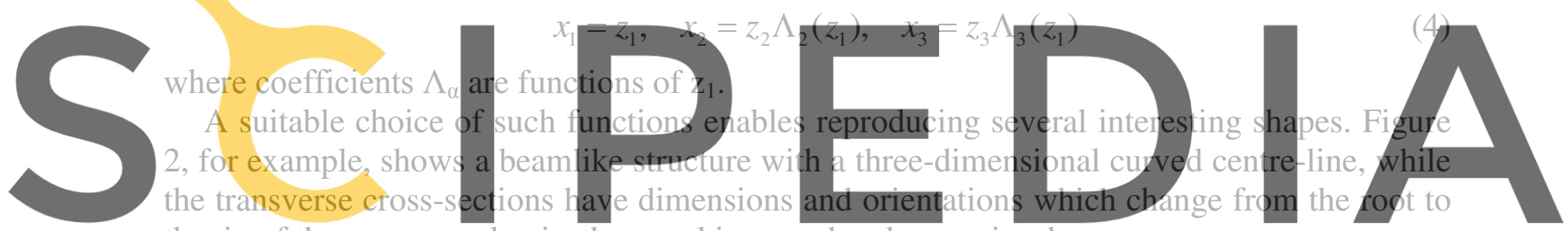

the tip of the structure, that is, they are bi-tapered and pre-twisted.

Register for free at https//www.scipedia.com to download the version without the watermark
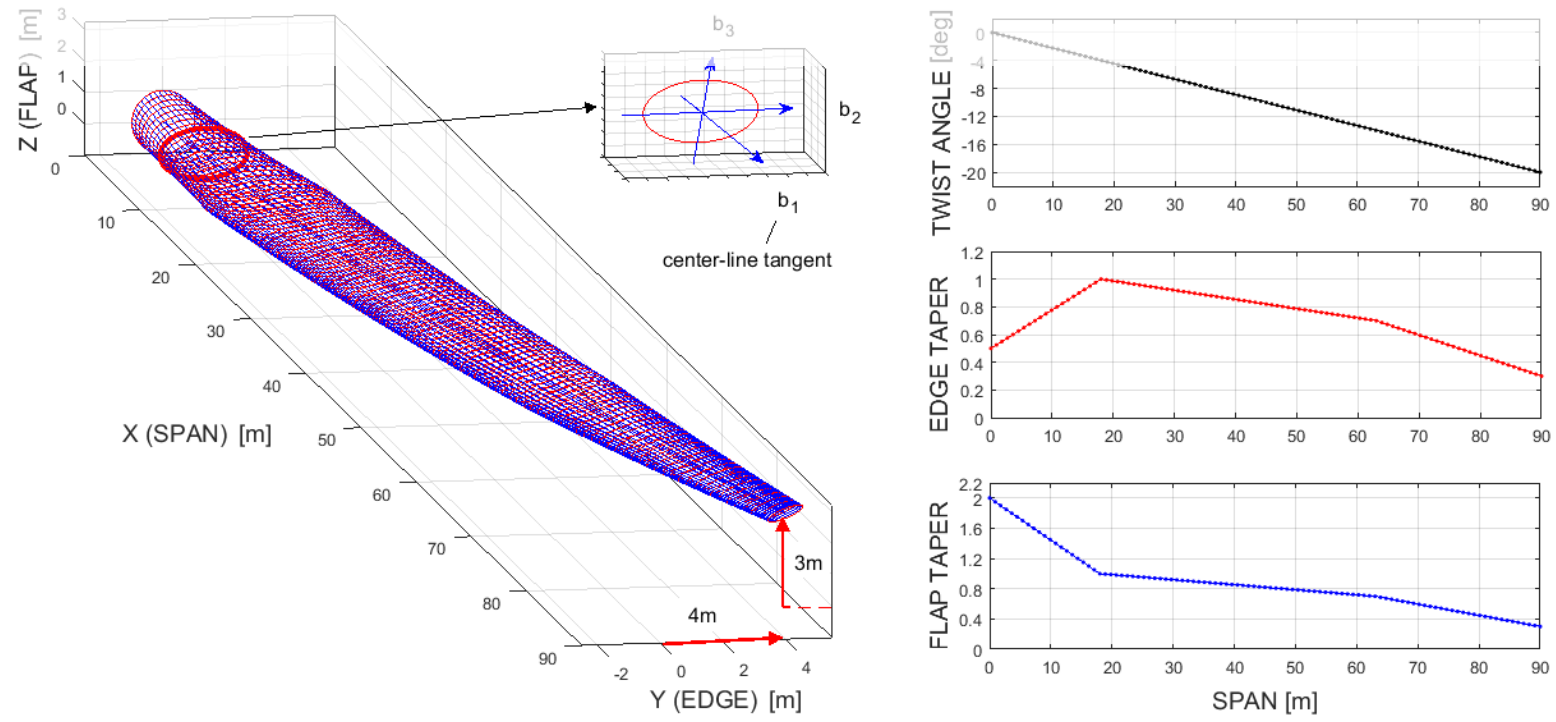

Figure 2: Example non-prismatic beamlike structure (left), and its twist and taper parameters (right) 
The position of the structure's points in the current state are defined in a similar manner by the (current) mapping function

$$
R_{A}\left(z_{i}, t\right)=R_{0 A}\left(z_{1}, t\right)+x_{\alpha}\left(z_{i}\right) a_{\alpha}\left(z_{1}, t\right)+w_{k}\left(z_{i}, t\right) a_{k}\left(z_{1}, t\right)
$$

where $\mathrm{R}_{0 \mathrm{~A}}$ provides the positions of the centre-line's points in the current state, while $\mathrm{w}_{\mathrm{k}}$ are the components of the 3D warping displacement with respect to $a_{k}$, introduced to describe the structure's deformed state without a-priori approximations.

We now introduce the kinematical variables we use to describe the motion of the structure, starting with the orthogonal tensor field $T$, which provides the relative orientation between $a_{i}$ and $b_{i}$, and the skew tensor fields $\mathrm{K}_{\mathrm{A}}$ and $\mathrm{K}_{\mathrm{B}}$, defined as follows

$$
T=A B^{T}, \quad K_{A}=A^{\prime} A^{T}, \quad K_{B}=B^{\prime} B^{T}
$$

The apex prime denotes the derivative with respect to the arc-length s. By combining equations (6), the following identity holds

$$
T^{T} T^{\prime}=T^{T} K_{A} T-K_{B}
$$

The left side of (7) defines a skew tensor field, hereafter denoted as $\mathrm{K}$. The corresponding axial vector, $\mathrm{k}$, can be determined by the relation

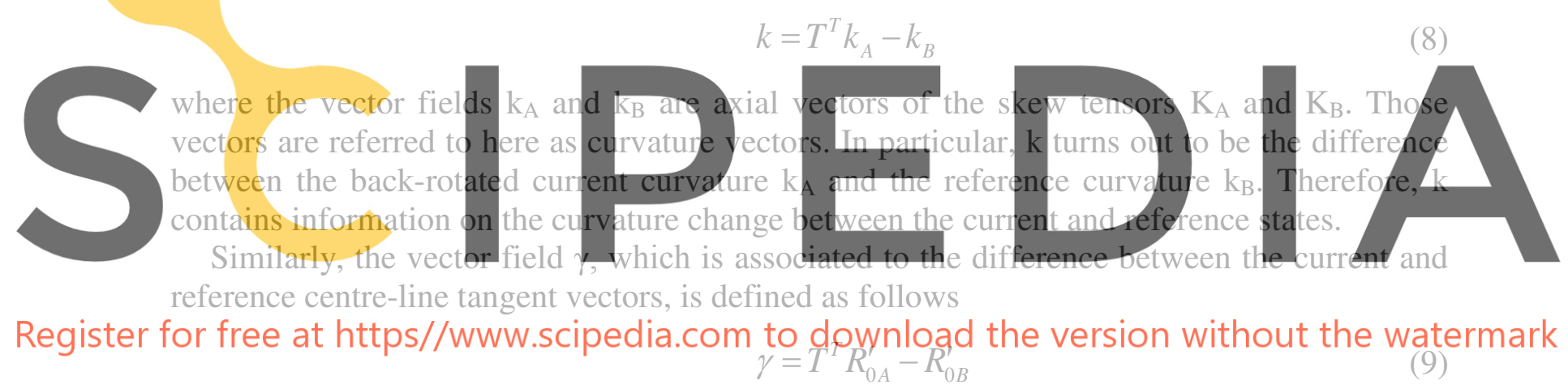

We also introduce the skew tensor fieid $\Omega$, whose axial vector is called $\omega$, which is related to the variation in the current local triad $a_{i}$ over the time $t$, as follows

$$
\Omega=A^{\cdot} A^{T}
$$

The apex dot denotes the derivative over time $t$. The local triad $b_{i}$ does not depend on time and so does function $\mathrm{R}_{0 \mathrm{~B}}$. On the contrary, $\mathrm{R}_{0 \mathrm{~A}}$ can change over time. Its variation is the time rate of change in the position of the current centre-line's points, $v_{0}$, that is

$$
R_{0 A}^{\cdot}=v_{0}
$$

By exploiting (6)-(11), we can also write kinematic relations which provide the time rate of vector fields $\gamma$ and $\mathrm{k}$ as functions of vector fields $\mathrm{v}_{0}$ and $\omega$, as follows

$$
\begin{aligned}
& v_{0}^{\prime}-\omega \wedge R_{0 A}^{\prime}=T \gamma^{\circ} \\
& \omega^{\prime}=T k^{.}
\end{aligned}
$$


where the operator $\wedge$ is the usual cross-product.

Hereafter $\gamma$ and $\mathrm{k}$ are also referred to as 1D strain measures, while the Green-Lagrange strain tensor E is referred to as 3D strain measure. Such tensor is moreover written in a form based on the assumptions of small warping and strain fields considered in this work. In particular, we assume that the reference dimension, $h$, of the cross-sections is much smaller that the characteristic length, $L$, of the centre-line (the beam is slender); the beam's curvatures are much smaller than $1 / h$; the warping fields, $w_{k}$, are considered small in the sense that their maximum order of magnitude is $h \varepsilon, \varepsilon<<1$ being a non-dimensional parameter, while the order of their derivative with respect to $z_{1}$ is at most $\varepsilon h / L$. In general, all components of the $1 \mathrm{D}$ and $3 \mathrm{D}$ strain measures are assumed to be small in the sense that their order of magnitude is at most $\varepsilon$. For the considered structure, the strain tensor E can be written in the form

$$
E \simeq \frac{T^{T} H+H^{T} T}{2}-I
$$

where tensor $\mathrm{H}$, which is the gradient of the current position $\mathrm{R}_{\mathrm{A}}$ with respect to the reference position $R_{B}$, is defined as follows

$$
H=\frac{\partial R_{A}}{\partial R_{B}}
$$

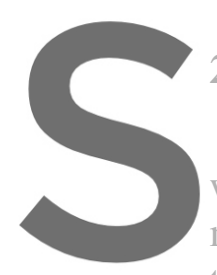

2.2 Stress measures and constitutive model
Given the strain tensor E, the corresponding stress fields in
when a constitutive model is chosen. Limiting our attentic
mechanical theory, in the case of small strain, the second (sy

tensor, $\mathrm{S}$, is expressed in terms of the Green-Lagrange strain tensor, E, as follows

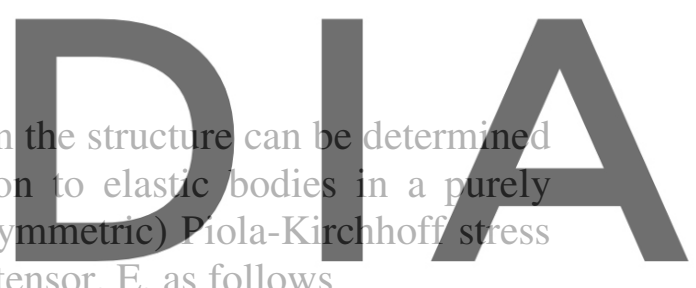

Register for free at https//www.scipedia.com to2downdodil the version without the watermark

where $\mu$ and $\lambda$ are known material parameters and I is the identity tensor [23].

We can now define the stress resultants on each transverse cross-section of the structure, in terms of force and moment resultants, $\mathrm{F}$ and $\mathrm{M}$, as follows

$$
\begin{aligned}
& F=\int_{\Sigma} P_{i 1} a_{i} \\
& M=\int_{\Sigma} x_{\alpha} P_{i 1} a_{\alpha} \wedge a_{i}
\end{aligned}
$$

In (16), $\Sigma$ is the domain corresponding to the cross-section on which integration is performed, the force and moment vector fields, $\mathrm{F}$ and $\mathrm{M}$, depend on arc-length $\mathrm{s}$, and the components $\mathrm{P}_{\mathrm{ij}}$ of the first Piola-Kirchhoff stress tensor, P, are defined as follows

$$
P_{i j}=P \cdot a_{i} \otimes b_{j}
$$

where symbol $\cdot$ is the usual scalar (or dot) product, symbol $\otimes$ is the usual tensor (or dyadic) product and, in the considered case, $\mathrm{P}=\mathrm{TS}$. In the present case it is moreover possible to write the classical Cauchy stress tensor, $\mathrm{C}$, as $\mathrm{C}=\mathrm{TST}^{\mathrm{T}}$. 


\subsection{Expended power and balance equations}

To complete the model formulation, we introduce the principle of expended power and the balance equations for the considered structure. First of all, we assume that the beamlike body studied in this paper is three-dimensional and hyper-elastic [23]. Its interactions with the external environment are quantified, for each velocity field attainable by the body, through the following linear functional of its velocity field, $\Pi_{e}$, called the external power

$$
\Pi_{e}=\int_{\partial V} p \cdot v+\int_{V} b \cdot v
$$

In (18), $b$ are body loads per unit reference volume $\mathrm{V}, p$ are surface loads per unit area of the reference boundary $\partial \mathrm{V}$, and $v$ is the referential description of the time rate of the current position of the body's points, given by

$$
v=v_{0}+\omega \wedge x_{\alpha} b_{\alpha}+w^{\cdot}
$$

where $w^{\circ}$ is the time rate of the warping displacement.

Interactions among different parts of the body are instead quantified by the internal power $\Pi_{\mathrm{i}}$, which is defined as follows

$$
\Pi_{i}=\frac{d}{d t} \int_{V} \Phi
$$

\section{where $\Phi$ is the energy $\mathrm{S}$ and $\mathrm{E}$ (i.e. $2 \Phi=\mathrm{S} \cdot \mathrm{E}$ \\ According to the \\ body, its interactions \\ value of the evolution $\mathrm{p}$
principle is a usual technic
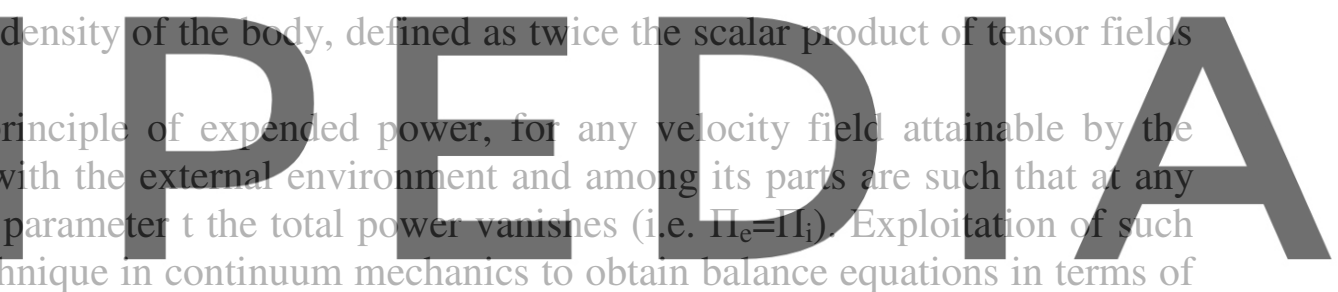 \\ the unknowns of the problem (see, e.g., [13] and [23]). In our case, it makes it possible to

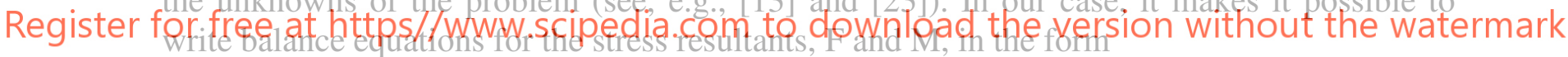

$$
\begin{aligned}
& F^{\prime}+F_{s}=0 \\
& M^{\prime}+R_{0 A}^{\prime} \wedge F+M_{s}=0
\end{aligned}
$$

where $F_{s}$ and $M_{s}$ are the resultants of the body and surface external loads per unit length of the reference centre-line. The same principle also enables writing balance equations to determine the warping fields, $\mathrm{w}_{\mathrm{k}}$, which govern the cross-sectional deformation. In particular, in the case in which the body loads and surface actions on the beam's lateral surface are neglected in calculating the warping fields, or vanish, it is possible to reduce the determination of the warping fields, $\mathrm{w}_{\mathrm{k}}$, to those that verify the following variational statement

$$
\delta \int_{V} \Phi=0
$$

where the symbol $\delta$ denotes the variation of the energy function with respect to the warping fields. Note that suitable warping fields satisfying (22) can be obtained via the corresponding Euler-Lagrange equations [24] by using numerical methods or, in particular cases, by means of analytical approaches providing closed-form results, as is discussed in the following. 


\subsection{Cross-sectional warping and centre-line deflection}

So far we have introduced the main ingredients of our modeling approach for the structures considered in this work, which are three-dimensional, beamlike, slender, and non-prismatic, and have defined the strain measures, stress measures, and balance equations we use to describe their mechanical behavior. By exploiting such ingredients, the resolution of the three-dimensional nonlinear elasticity problem for the aforementioned structures is now reduced to the solution of two main problems: the first governs the cross-sectional warping motion and its strong formulation can be given in terms of partial differential equations (PDEs) defined over a reference bi-dimensional domain (as is further discussed in the following section). The second problem governs the centre-line motion and can be expressed in terms of a set of nonlinear ordinary differential equations (ODEs) defined over a reference line (as in [18], for instance).

Specifically, in the following we exploit condition (22) to obtain the PDEs problem the solution of which enables determining the warping of the transverse cross-sections (and show a case in which we can obtain analytical closed-form solutions). The displacements of the centre-line's points are instead determined by solving the nonlinear ODEs problem based on the balance equations (21) for the stress resultants and the corresponding kinematic and constitutive relations introduced in the previous sections 2.1-2.3. Such nonlinear problem is integrated (numerically) with respect to arc-length s.
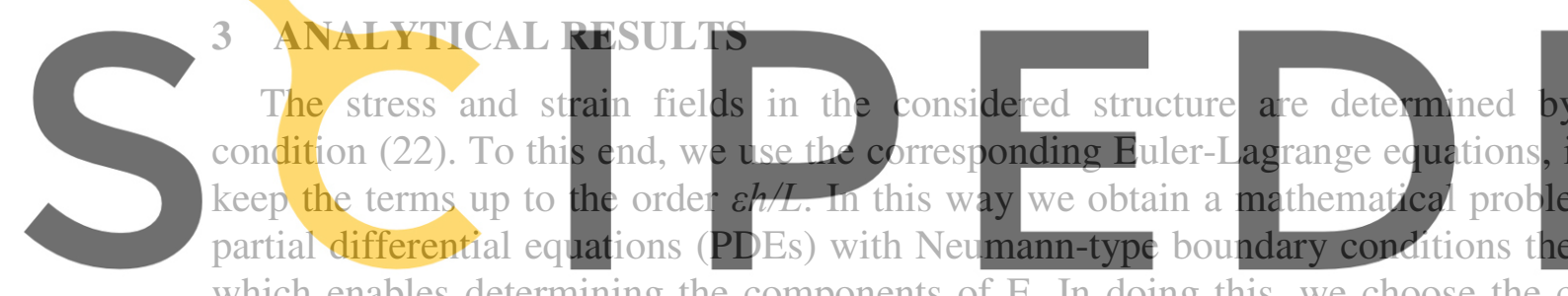

which enables determining the components of E. In doing this, we choose the current local

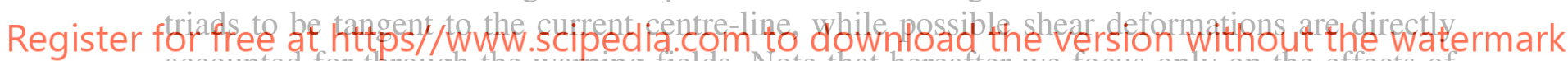
accounted for through the warping fields. Note that hereafter we focus only on the effects of

the cross-sectional taper on the stress and strain fields; other geometric effects, such those related to the cross-sectional pre-twist, are not considered.

Proceeding in this way, the components $\mathrm{E}_{11}, \mathrm{E}_{21}$ and $\mathrm{E}_{31}$ of tensor $\mathrm{E}$, associated to the outof-plane deformation of the transverse cross-sections, can be written in the form

$$
\begin{aligned}
& E_{11}=k_{2} x_{3}-k_{3} x_{2}+\gamma_{1}+e_{1,1} \\
& 2 E_{21}=e_{1,2}-k_{1} x_{3}+2(1+v)\left(k_{2} x_{3}-k_{3} x_{2}+\gamma_{1}\right) \Lambda_{2}^{-1} \Lambda_{2}^{\prime} x_{2}+e_{2} \\
& 2 E_{31}=e_{1,3}+k_{1} x_{2}+2(1+v)\left(k_{2} x_{3}-k_{3} x_{2}+\gamma_{1}\right) \Lambda_{3}^{-1} \Lambda_{3}^{\prime} x_{3}+e_{3}
\end{aligned}
$$

where $E_{i j}=E \cdot b_{i} \otimes b_{j}, v$ is Poisson's ratio, the subscript comma denotes the derivative with respect to $\mathrm{x}_{\mathrm{i}}$, and the scalar fields $\mathrm{e}_{1}, \mathrm{e}_{2}, \mathrm{e}_{3}$ can be obtained by solving the PDEs problems

$$
\begin{aligned}
& e_{1,22}+e_{1,33}=0 \text { in } \Sigma \\
& \left(e_{1,2}-k_{1} x_{3}\right) n_{2}+\left(e_{1,3}+k_{1} x_{2}\right) n_{3}=0 \quad \text { on } \partial \Sigma
\end{aligned}
$$




$$
\begin{array}{ll}
e_{2,2}+e_{3,3}=a_{2} x_{2}+a_{3} x_{3} & \text { in } \Sigma \\
e_{3,2}-e_{2,3}=b_{2} x_{2}+b_{3} x_{3} & \text { in } \Sigma \\
e_{2} n_{2}+e_{3} n_{3}=0 \quad \text { on } \partial \Sigma &
\end{array}
$$

In (24)-(25), $\Sigma$ and $\partial \Sigma$ are the cross-sectional domain and its boundary, $\mathrm{n}_{\alpha}$ are components of the outward unit normal vectors on $\partial \Sigma$, and coefficients $a_{\alpha}$ and $b_{\alpha}$ are linear functions of the strain measures $\mathrm{k}_{\alpha}$ and their $s$-derivative. Such coefficients, which also depend on the beam's initial shape through the taper coefficients $\Lambda_{2}$ and $\Lambda_{3}$, are given by

$$
\begin{aligned}
& a_{2}=+2(1+v) k_{3}^{\prime}+2(1+v)\left(\Lambda_{3}^{-1} \Lambda_{3}^{\prime}+2 \Lambda_{2}^{-1} \Lambda_{2}^{\prime}\right) k_{3} \\
& a_{3}=-2(1+v) k_{2}^{\prime}-2(1+v)\left(\Lambda_{2}^{-1} \Lambda_{2}^{\prime}+2 \Lambda_{3}^{-1} \Lambda_{3}^{\prime}\right) k_{2} \\
& b_{2}=-2 v k_{2}^{\prime}-2(1+v) \Lambda_{2}^{-1} \Lambda_{2}^{\prime} k_{2} \\
& b_{3}=-2 v k_{3}^{\prime}-2(1+v) \Lambda_{3}^{-1} \Lambda_{3}^{\prime} k_{3}
\end{aligned}
$$

The approach used can also provide relations for the components $E_{22}, E_{33}$, and $E_{23}$ of $E$, related to the in-plane deformation of the transverse cross-sections, plus the relevant PDEs problem. It is worth noting that the components of the stress resultants (16) depend on the components $\mathrm{E}_{11}, \mathrm{E}_{21}, \mathrm{E}_{31}$ of $\mathrm{E}$, and the additional term $\mathrm{E}_{\mathrm{SV}}=\mathrm{E}_{22}+\mathrm{E}_{33}+2 v \mathrm{E}_{11}$, which is related to the aforementioned in-plane deformations. However, we also note that in the present case $E_{S V}$ is of higher order with respect to other terms in the expressions of the stress resultants [18],

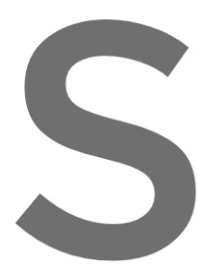
and vanishes for prism problem related to the cross-sectional taper in and strain fields in the

3.1. The case of the bi-tapered ellipticall cross-sections
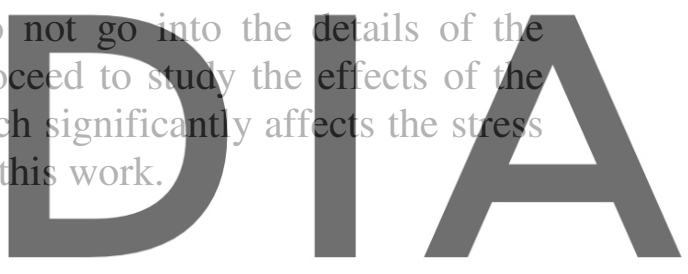

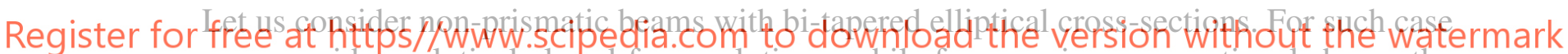
we can provide analytical closed-form solutions, while for generic cross-sectional shapes the

PDEs problems introduced in the foregoing have to be solved with the aid of numerical methods. However, this is not surprising. In fact, analytical solutions are available only for a limited number of cases even in the classical linear theory of prismatic beams [4].

In this case, problems (24)-(25) can be solved without resorting to numerical methods. In particular, we obtain the following analytical results

$$
\begin{aligned}
& e_{1}=\frac{d_{3}^{2}-\rho^{-2} d_{2}^{2}}{d_{3}^{2}+\rho^{-2} d_{2}^{2}} k_{1} x_{2} x_{3} \\
& e_{2}=+\frac{c_{\alpha} x_{\alpha} x_{3}}{d_{3}^{2} \Lambda_{3}^{2}}+\frac{c_{3}+a_{2} d_{2}^{2} \Lambda_{2}^{2}}{2}\left(\frac{x_{2}^{2}}{d_{2}^{2} \Lambda_{2}^{2}}+\frac{x_{3}^{2}}{d_{3}^{2} \Lambda_{3}^{2}}-1\right) \\
& e_{3}=-\frac{c_{\alpha} x_{\alpha} x_{2}}{d_{2}^{2} \Lambda_{2}^{2}}-\frac{c_{2}-a_{3} d_{3}^{2} \Lambda_{3}^{2}}{2}\left(\frac{x_{2}^{2}}{d_{2}^{2} \Lambda_{2}^{2}}+\frac{x_{3}^{2}}{d_{3}^{2} \Lambda_{3}^{2}}-1\right)
\end{aligned}
$$

In (27), $d_{2}$ and $d_{3}$ are the major semi-axes of a reference elliptical cross-section (e.g. the one at the root section), while coefficients $c_{2}$ and $c_{3}$ are defined as follows 


$$
\begin{aligned}
& c_{2}=\left(b_{2}+\rho^{2} a_{3}\right)\left(1+3 \rho^{2}\right)^{-1} \Lambda_{3}^{2} d_{3}^{2} \\
& c_{3}=\left(b_{3}-\rho^{-2} a_{2}\right)\left(1+3 \rho^{-2}\right)^{-1} \Lambda_{2}^{2} d_{2}^{2}
\end{aligned}
$$

where $\rho=\Lambda_{3} / \Lambda_{2}$ is a known function of $z_{1}$. Using such results, we can also calculate the strain fields in the structure (23), and the corresponding stress fields. An important result is that the effects of the cross-sectional taper appear explicitly in all above equations in terms of two application-oriented functions, i.e. the taper coefficients $\Lambda_{2}$ and $\Lambda_{3}$. It is also worth noting that the model and results presented so far generalize those of the linear theory of prismatic beams $[4,23]$ and reduce to the them for prismatic beams undergoing small displacements.

Unfortunately, analytical solutions to problems (24)-(25), like those shown here (27), can be obtained only for a few cases. However, such problems can always be solved with the aid of numerical methods for all other cases as well.

\section{NUMFRICAI EXAMPI FS}

In this section we show the results obtainable via the modeling approach introduced in the previous sections. The model has been implemented in a numerical code written in the Matlab language, referred to as 3D-BLM. The results from 3D-BLM in terms of displacement, strain and stress fields are compared with those from 3D-FEM simulations performed with Ansys to show the computational efficiency and accuracy of the proposed approach.

In the test case reported here, we consider a straight beam, with bi-tapered elliptical crosssections, undergoing large displacements. The reference transverse eross-section at the rogt
has its major semi-axes equal to $\mathrm{d}_{2}=2 \mathrm{n}$ (edgewise) and $\mathrm{d}_{3}=2 \mathrm{~m}$ (flapwise). The dimensions of
the other cross-sections reduce from the root to the tip, with linear reduction edgewise (equal
to $50 \%$ at the tip) and parabolic reduction flapwise ( $85 \%$ at the tip). The material propdrties
are described in terms of reference values of Young's modulus, 70GPa. and Poisson's ratio,
0.25. The structure is fixed at one end (the root), and loaded at the other (the tip) by a flapwise force, F, of progressively increasing magnitude, as shown in Figure 3 (left).

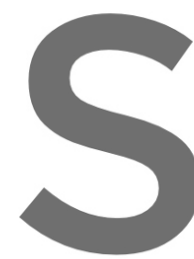

Register for free at https//www.scipedia.com to download the version without the watermark

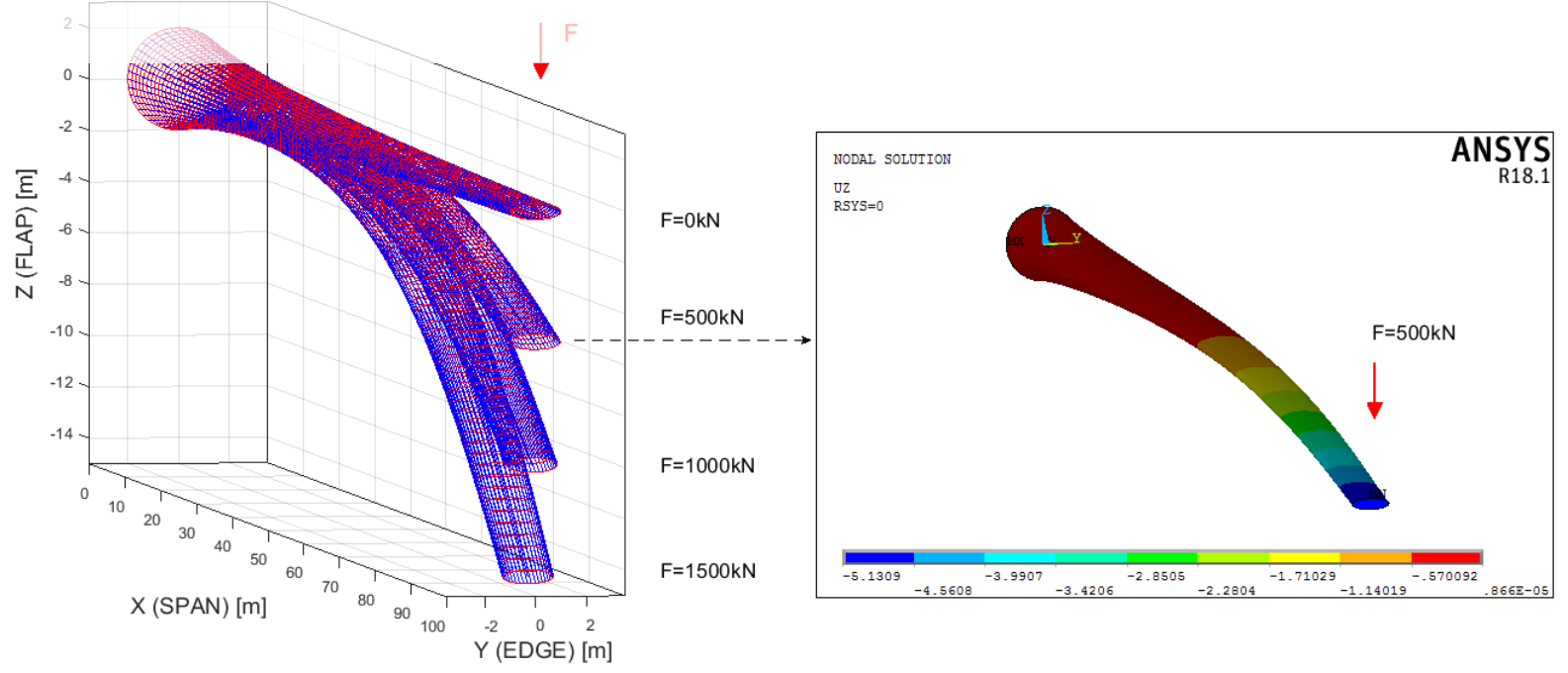

Figure 3: Global deflection with 3D-BLM for increasing F (left) and 3D-FEM for F=500kN (right) 
The results obtained from 3D-BLM, and those given by nonlinear 3D-FEM simulations, are now summarized. Specifically, Figure 3 (left) shows the structure's un-deformed shape $(\mathrm{F}=0$ ), its deformed shapes obtained from $3 \mathrm{D}-\mathrm{BLM}$ for $\mathrm{F}=500 \mathrm{kN}, \mathrm{F}=1000 \mathrm{kN}, \mathrm{F}=1500 \mathrm{~N}$ (left), and the deformed shape given by $3 \mathrm{D}-\mathrm{FEM}$ for $\mathrm{F}=500 \mathrm{kN}$ (right). Figure 4 , instead, presents comparisons in terms of tip-displacements (left) and simulation times (right), which show the computational efficiency and accuracy of the proposed modeling approach.
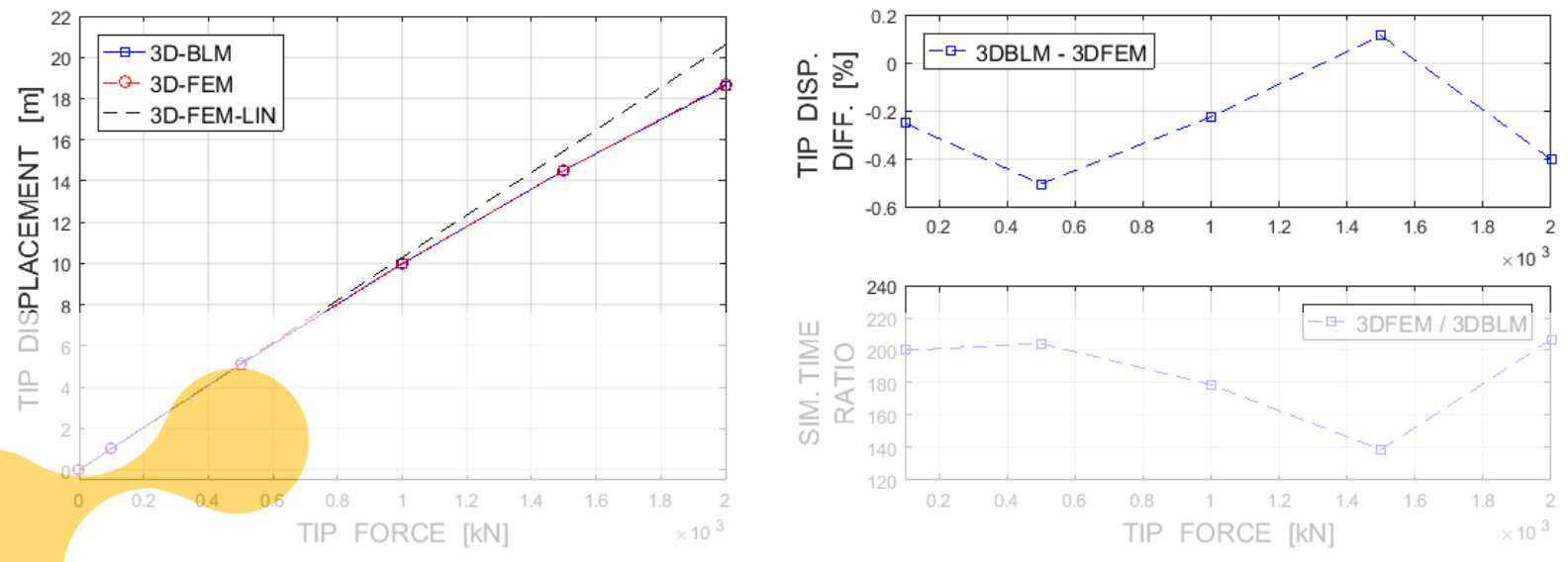

Figure 4: Comparison of tip-displacements (left), tip-displacement differences and simulation times (right)

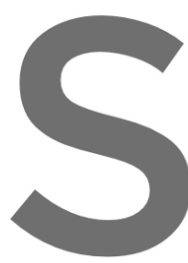

Apart from such resul information, such as th strain fields (13), and th

By way of example those obtained from no
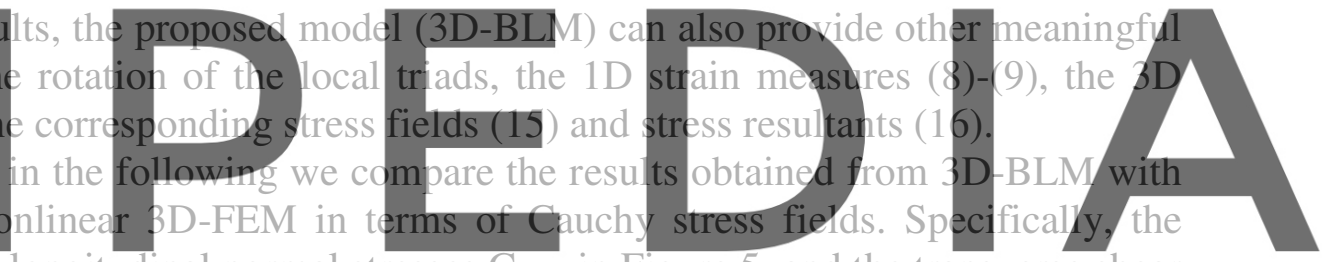

comparisons are for the longitudinal normal stresses $\mathrm{C}_{X X}$, in Figure 5, and the transverse shear

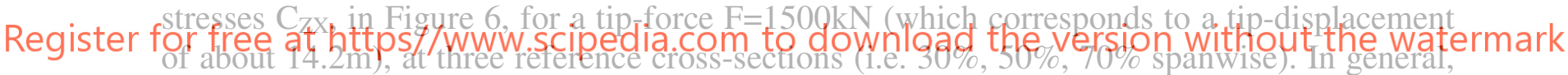

we have observed that the normal stresses follow a Navier-like distribution in the transverse

cross-sections (they are almost linear in $\mathrm{x}_{3}$ ), while the transverse shear stress distributions are quite different from those predictable by the linear theory of prismatic beams. In fact, the shear stresses in non-prismatic beams do not generally vanish at the cross-section's boundary and their distributions can change from cross-section to cross-section (i.e. spanwise).
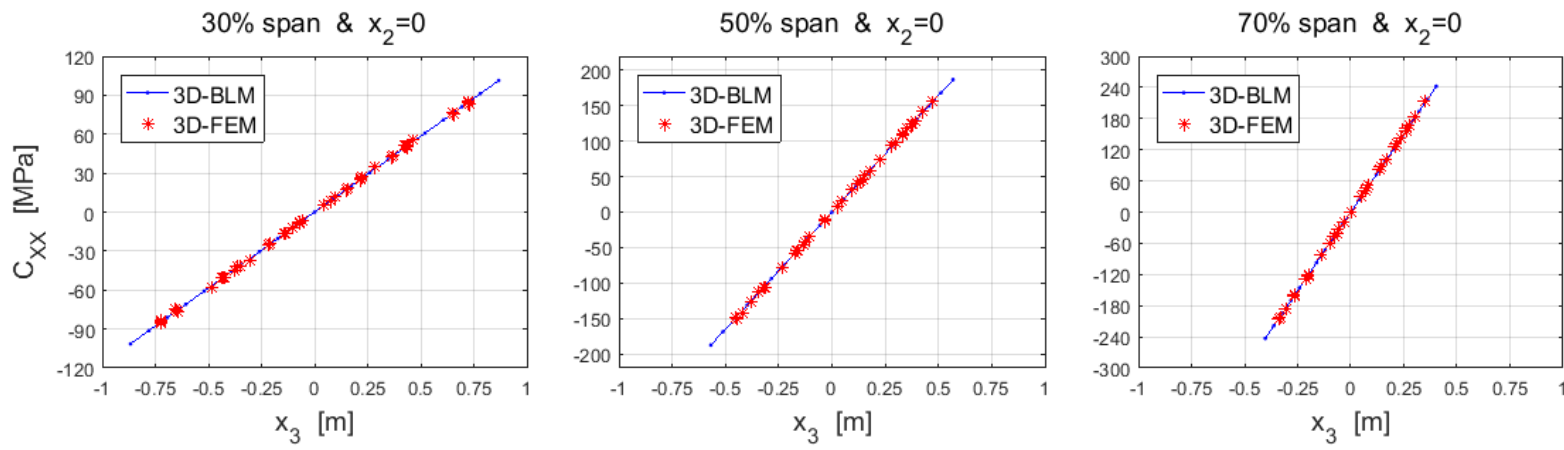

Figure 5: Comparison of longitudinal stress $C_{X X}$ in cross-sections at $30 \%, 50 \%, 70 \%$ span, for $F=1500 \mathrm{kN}$ 

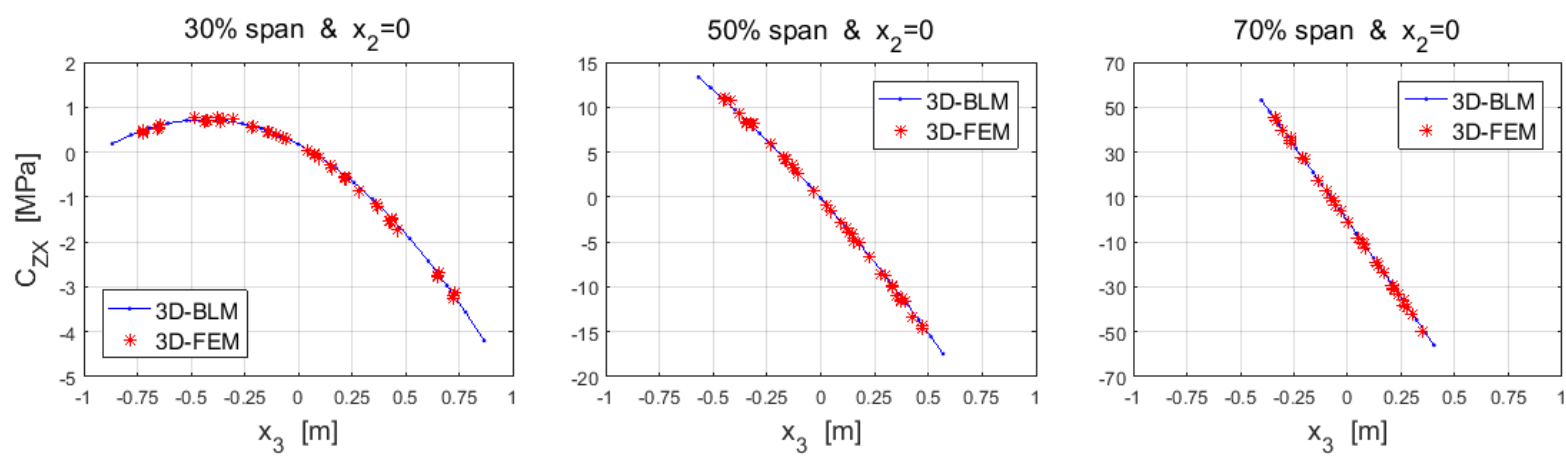

Figure 6: Comparison of transverse shear stress $\mathrm{C}_{\mathrm{ZX}}$ in cross-sections at $30 \%, 50 \%, 70 \%$ span, for $\mathrm{F}=1500 \mathrm{kN}$

Similar results have been obtained for other cross-sections and tip-forces, as well as for other geometries (see, e.g., [18]). In all cases the results obtained confirm the computational efficiency and accuracy of the proposed approach compared to nonlinear 3D-FEM.

The proposed model can thus be used to predict the mechanical behavior of non-prismatic beamlike structures as those considered in this work, which may undergo large displacements of the centre-line's points, warping of the transverse cross-sections in and out of plane, and small strains, as it can furnish accurate information on the deformed states of such structural elements in terms of displacement, strain and stress fields.

\section{CONCLUSIONS}

Many complex engineering structures, e.g. blades of wind turbines and helicopters, are beamlike and non-prismatic. Their mechanical behavior can be simulated by exploiting 3D beam models which are computationally efficient, accurate, and explicitly consider the main geometric design features of such structures, the large deflections of their centre-line and the 3D warping of their transverse cross-sections. In this work, non-prismatic beamlike structures have been modeled analytically. Their main geometric features (e.g. the cross-sectional taper) have been explicitly included in the model. The approach presented has been shown to be suitable for large deflections of the centre-line and small warping of the cross-sections. The 3D strain tensor has been calculated analytically in terms of the structure's geometric parameters, 1D strain measures and 3D warping fields. A variational approach has been exploited to obtain suitable warping fields. The analytical results obtained for bi-tapered beams, which generalize those of the linear theory of prismatic beams, have been presented and compared to the results of nonlinear 3D-FEM analyses. The results presented confirm the effectiveness of the modeling approach and show the information it can provide.

\section{REFERENCES}

[1] Ashwill T.D., Kanaby G., et al, Development of the swept twist adaptive rotor (STAR) blade, 48th AIAA Aerospace sciences meeting, Orlando, FL, Jan. 4-7, 2010.

[2] Bak C., Zahle F., et al., Description of the DTU 10MW reference wind turbine, DTU Wind Energy Report-I-0092, Denmark, 2013.

[3] Wang L., Liu X., Kolios A., State of the art in the aeroelasticity of wind turbine blades: aeroelastic modelling, Renewable and sustainable energy review, 64, 195-210, 2016.

[4] Love A.E.H., A treatise on the mathematical theory of elasticity, 4th ed., Dover 
Publications, NY, 1944.

[5] Timoshenko S.P., Goodier J.N., Theory of elasticity, 2nd ed., McGraw-Hill, 1951.

[6] Reissner E., On finite deformation of space curved beams, Journal of applied mathematics and physics, 32, 734-744, 1981.

[7] Hodges D.H., Geometrically exact equations for beams, Encyclopedia of Continuum Mechanics, Springer Verlag, Germany, 2018.

[8] Antman S.S., Warner W.H., Dynamical theory of hyper-elastic rods, Arch. Rational Mech. Anal., 23, 135-162, 1966.

[9] Rubin M.B., Cosserat theories: shells, rods and points, Solid mechanics and its applications, Springer Netherlands, 1st ed., 2000.

[10] Simo J.C., A finite strain beam formulation, the three-dimensional dynamic problem, part I, Computer methods in applied mechanics and engineering, 49, 55-70, 1985.

[11] Berdichevsky V.L., On the energy of an elastic rod, Journal of Applied Mathematics and Mechanics, 45, 518-529, 1981.

[12] Yu W., Hodges D.H., Ho J.C., Variational asymptotic beam-sectional analysis - an updated version, International journal of engineering science, 59, 40-64, 2012.

[13] Ruta G., Pignataro M., Rizzi N., A direct one-dimensional beam model for the flexuraltorsional buckling of thin-walled beams, Journal of Mechanics of materials and structures, 1, 1479-1496, 2006.

[14] Kunz D.L., Survey and comparison of engineering beam theories for helicopter rotor blades, J. of Aircraft, 31, 473-479, 1994.

[15] Rafiee M., Nitzsche F., et al., Dynamics, vibration and control of rotating composite beams and blades: a critical review, Thin-walled structures, 119, 795-819, 2017.

[16] Rosen A., Structural and dynamic behavior of pre-twisted rods and beams, American Society of Mechanical Engineers, 44, 483-515, 1991.

[17] Migliaccio G., Ruta G, et al., Curved and twisted beam models for aeroelastic analysis of wind turbine blades in large displacements, XXIV AIMETA conference, 15-19 Sept. 2019, Rome, Italy, Lecture notes in mechanical engineering, Springer, 2020.

[18] Migliaccio G., Ruta G., et al., Beamlike models for the analyses of curved, twisted and tapered horizontal-axis wind turbine (HAWT) blades undergoing large displacements, Wind Energy Science, 5, 685-698, https://doi.org/10.5194/wes-5-685-2020, 2020.

[19] Pai P.F., Three kinematic representations for modeling of high flexible beams and their applications, International Journal of solids and structures, 48, 2764-2777, 2011

[20] Danielson D.A., Hodges D.H., Nonlinear beam kinematics by decomposition of the rotation tensor, Journal of applied mechanics, 54, 258-262, 1987.

[21] Argyris J., An excursion into large rotations, Computer methods in applied mechanics and engineering, 32, 85-155, 1982.

[22] Migliaccio G., and Ruta G., Rotor blades as curved, twisted, and tapered beam-like structures subjected to large deflections, Engineering Structures, 222:111089, 2020.

[23] Gurtin M.E., An introduction to continuum mechanics, Mathematics in science and engineering, Academic Press, 1st ed., 1981.

[24] Courant R., Hilbert D., Methods of mathematical physics, Interscience Publisher, 1st ed., 1953. 